\title{
A Three-Dimensional Functional Assessment of Heart and Vessel Development in the Larva of the Zebrafish (Danio rerio)
}

\author{
Brian Bagatto ${ }^{1, *}$ \\ Warren Burggren ${ }^{2}$ \\ ${ }^{1}$ Department of Biology, University of Akron, Akron, Ohio \\ 44325; ${ }^{2}$ Department of Biological Sciences, University of \\ North Texas, Denton, Texas 76203
}

Accepted 5/24/2005; Electronically Published 11/7/2005

\begin{abstract}
There has been considerable recent interest in the development of the circulation in the zebrafish. Optical techniques typically used to visualize changes in heart size allow measurement of stroke volume during early vertebrate development, but this approach is complicated in zebrafish larvae because of the heart's irregular shape and its significant change in morphology during the first $6 \mathrm{~d}$ of development. By use of a threedimensional integration of the early zebrafish heart and vessels, we have greatly reduced measurement error of stroke volume and cardiac output and have determined the cross-sectional growth of major vessels in the developing zebrafish larvae. A dramatic $500 \%-600 \%$ increase in cardiac output (from 10 to

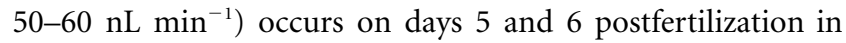
Danio rerio. Cross-sectional area of key vessels (dorsal artery, caudal artery, dorsal vein) as well as between-individual variation significantly decreased over the first $6 \mathrm{~d}$ of development. Associated with the decrease in cross-sectional area is a significant increase in red blood cell velocity on days 5 and 6 postfertilization. Together, the three-dimensional data of the cardiac and vascular systems have shown that the most profound physiological and developmental changes occur in days 5 and 6 , which corresponds with the appearance of the adult form of the heart and the transition from diffusive to convective $\mathrm{O}_{2}$ supply to internal tissues.
\end{abstract}

\section{Introduction}

The cardiovascular system is the first organ system to become fully functional in the vertebrate embryo, and its development

\footnotetext{
*Corresponding author; e-mail: bagatto@uakron.edu.
}

Physiological and Biochemical Zoology 79(1):194-201. 2006. (C) 2006 by The University of Chicago. All rights reserved. 1522-2152/2006/7901-4125\$15.00 occurs in a similar way in all vertebrates (Fishman and Chien 1997; Glickman and Yelon 2002). Consequently, the function of the early heart has long been a source of investigation. In many embryos and larvae, cardiovascular function can be measured optically through video analysis of volume changes of the beating heart (Burggren and Fritsche 1995; Hou and Burggren 1995; Fritsche and Burggren 1996; Mirkovic and Rombough 1998; Burggren 2004). In zebrafish, the relative transparency of the larva allows an even more in-depth optical analysis of the vascular system. The integration of regional blood flow distribution, determined by tracking the movement of individual red blood cells, provides insights into regulation of peripheral resistance. For example, perfusion of the gut could be quantified as the zebrafish switches from using yolk to absorbing nutrients across the intestinal wall. Such experiments allow the clear differentiation of active vasomotor responses from intrinsic cardiac changes (Schwerte and Pelster 2000; Schwerte and Fritsche 2003; Bagatto 2005).

Though powerful in probing the developing cardiovascular system, video analysis of the zebrafish embryo has inherent problems. For example, an optical analysis of the beating heart is performed in a planar field with two dimensions, yet the vertebrate heart develops asymmetrically in three dimensions. This becomes problematic when optically assessing the zebrafish's primitive heart tube, which forms by $22 \mathrm{~h}$ postfertilization (hpf; at $28.5^{\circ} \mathrm{C}$ ), just before the heart begins beating by $24 \mathrm{hpf}$ (Stainier and Fishman 1992). By $30 \mathrm{hpf}$, the heart tube starts to loop to the right side of the embryo, and by $36 \mathrm{hpf}$, chamber boundaries are evident (Stainier and Fishman 1992). By $60 \mathrm{hpf}$, cardiac valves are present, and by the fifth day, the heart has assumed its adult configuration, with the atrium sitting dorsally with respect to the ventricle (Weinstein and Fishman 1996). Thus, in the zebrafish, as little as $1 \mathrm{~h}$ of development or a slight variation in video recording angle can result in significant differences in measured stroke volume and calculated cardiac output. In fact, the commonly used left lateral view of the heart in combination with a prolate spheroid model of the heart simply does not allow accurate measurement of stroke volume and thus cardiac output during early development of zebrafish (Burggren and Fritsche 1995).

To overcome the limitations of two-dimensional optical analysis of the circulation, this study uses an integrated threedimensional view of the larval zebrafish heart. This approach has allowed more accurate measurements of stroke volume and calculation of cardiac output as well as cross-sectional area of major vessels. We show the utility of these techniques by testing 
the hypothesis that red blood cell velocity changes in complex patterns as vessels increase in diameter as the adult form of the heart nears completion.

\section{Material and Methods}

Animals

Adult long-fin gold zebrafish, Danio rerio, were obtained from Scientific Hatcheries (Huntington Beach, CA) and were used as breeding stock to produce the embryos and larvae used in this study. Long-fin gold zebrafish develop with virtually no pigment, which allows the heart and vasculature to be viewed through the body wall for up to $40 \mathrm{~d}$ postfertilization (compared with approximately $3 \mathrm{~d}$ for wild stock). Standard husbandry procedures were followed according to Westerfield (1995), and breeding procedures were followed according to Bagatto et al. (2001). A 14L:10D cycle was used, and the experimental temperature was $25^{\circ} \mathrm{C}$. Fecundity of zebrafish stocks from Scientific Hatcheries is significantly improved at $25^{\circ} \mathrm{C}$, as opposed to at the published standard temperature of $28.5^{\circ} \mathrm{C}$ (B. Bagatto and W. Burggren, unpublished data).

\section{Imaging Techniques}

1. Immobilization of Larvae. All imaging reported in this study required immobilization of the living larva. Unanesthetized zebrafish larvae were live mounted in 1\% agarose (SeaPlaque; gelling temperature $=26^{\circ}-30^{\circ} \mathrm{C}$ ) and placed in a temperaturecontrolled environment on the stage of the inverted microscope for video recording (Schwerte and Pelster 2000). Briefly, this process involved liquification of the agarose with gentle heating followed by cooling to $30^{\circ} \mathrm{C}$. The agarose was pipetted into a 4-mm-wide glass trough. A zebrafish larva was then immersed in the liquid agarose. The larva was gently positioned with a blunt probe to orient parallel to the cylinder length, and once the larva was in place, the cylinder was placed against an ice pack to quickly solidify the agarose. The mold was removed from the glass trough and placed into a temperature-controlled bath set at $25^{\circ} \mathrm{C}$. Measurements of oxygen concentrations within the agarose show that conditions go hypoxic at approximately $5 \mathrm{~min}$ in day 6 larvae (B. Bagatto and W. Burggren, unpublished data). All measurements in this study were completed within 3 min of the larva's initial submergence into the agarose.

2. Image Capturing. The imaging techniques developed in this study are based on a standard set of techniques used by Hou and Burggren (1995) in Xenopus laevis and refined by Schwerte and Pelster (2000) for the zebrafish. The imaging system consisted of a trinocular inverted microscope (Nikon Eclipse TS100) equipped with a CCD black and white high-resolution camera. The camera was connected to the luminance input of an SVHS video recorder (Panasonic AG-7350). Images from the camera and video recorder were digitized using a framegrabber card (Flashbus MV) associated with a Pentium III 800 $\mathrm{MHz}$ computer. Live video was also digitized at 30 frames $\mathrm{s}^{-1}$ using video capturing hardware and associated software (Matrox G400 video display card and Rainbow Runner video capture card).

3. Two-Dimensional Analysis. Heart rate and stroke volume (required for cardiac output calculation) were determined by first capturing digitized video sequences of the ventricle of the immobilized larva. Heart rate was then calculated from the time interval required for 30 consecutive heartbeats. The perimeter of the ventricle image during end systole (a single frame selected from the cardiac cycle video) and during end diastole was outlined manually using a computer mouse (Hou and Burggren 1995; Schonweger et al. 2000). The perimeter was fitted with an ellipse (using ImagePro imaging software; Media Cybernetics, Silver Springs, MD), and major and minor axes measurements were extracted and exported to an Excel spreadsheet. Calculation of ventricle volume during systole and diastole was based on the formula for the volume of a prolate spheroid:

$$
\frac{4 \pi a b^{2}}{3}
$$

where $a$ represents the longitudinal axis of the ellipse and $b$ represents the width of the ellipse. The standard protocol involved analyzing six systoles and six diastoles from each animal to minimize within-animal variation and experimental measurement error. Stroke volume was calculated by subtracting mean systolic ventricular volume from mean diastolic ventricular volume. Cardiac output was calculated by multiplying stroke volume by mean heart rate for the given measurement time interval.

4. Three-Dimensional Analysis. Three-dimensional analysis required visualization of the heart and vessels from multiple angles. Consequently, a system was designed to rotate the living larva for optical measurements. Zebrafish embryos and larvae were immobilized longitudinally in agarose in the glass trough (see above). The agarose mold could be rotated, and video images of the zebrafish were captured through a $180^{\circ}$ ventrolateral rotation (see Fig. 1). This permitted diameter of a given vessel to be obtained at different angles $\left(0^{\circ}\right.$ [left lateral view], $45^{\circ}, 90^{\circ}$ [ventral view], $135^{\circ}$, and $180^{\circ}$ [right lateral view]) by the established methods described below. Each vessel diameter was represented as a straight line drawn using ImagePro. Vessel diameters obtained at $0^{\circ}$ and $180^{\circ}$ from the same larva were averaged, and the resulting line was plotted vertically using ImagePro. The line representing the vessel diameter at $90^{\circ}$ was drawn horizontally and centered on the previous vertical line. The lines constructed from $45^{\circ}$ and $135^{\circ}$ diameter measure- 


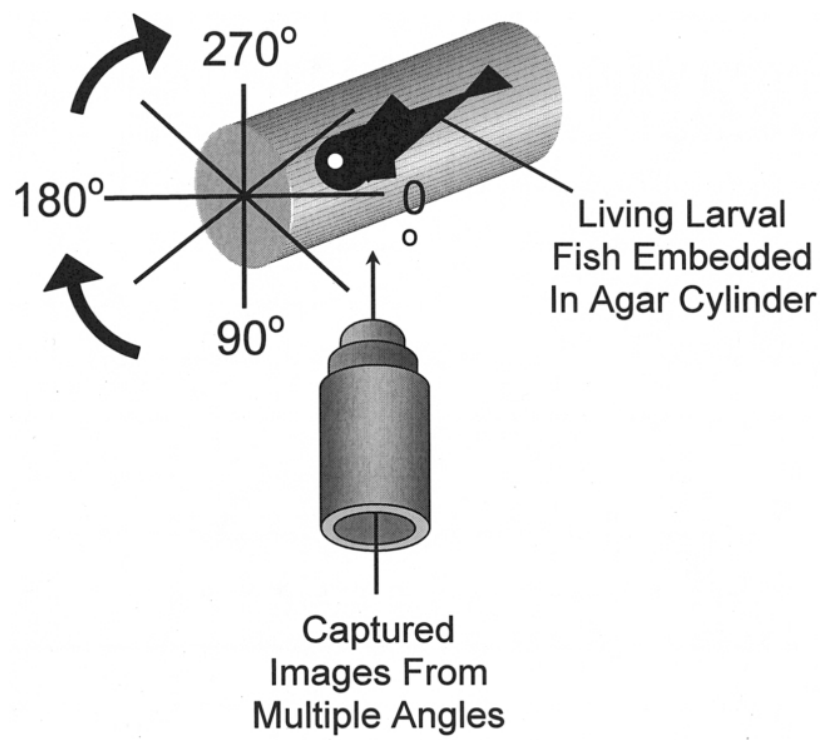

Figure 1. Schematic of larval zebrafish mounted in agarose and rotated to capture images at various angles.

ments were then placed and centered at their respective angles from the $0^{\circ} / 180^{\circ}$ line. The result of intersecting all of the measured diameters is an asterisk. The asterisk was then outlined using ImagePro and the resulting cross-sectional area calculated.

5. Cardiac Output Measurement. Rotation of the larva also allowed in vivo visualization of the cardiac cycle from the lateral as well as the ventral side. Previous studies assumed the width of the ventral side to be the same as the height of the lateral side. However, our empirical observations have led to a more accurate formula for calculating ventricular volume:

$$
\frac{8 A_{90} A_{0,180}}{3 \pi L}
$$

where $A_{90}$ is the area of the ventricle viewed on the ventral side, $A_{0,180}$ is the mean area of the traced ventricle on both lateral sides, and $L$ is the mean major axis length shared by all views. As indicated above, the means of the best end-systole and enddiastole frames were used in determining the mean stroke volume for a particular larva.

6. Vessel Diameter Measurement. Theoretical specifics of video analysis of red blood cells and digital visualization of the vessels have been thoroughly discussed by Schwerte and Pelster (2000). Briefly, a frame from an interlaced standard CCD video camera consists of two fields, odd and even. Images of moving red blood cells reveal a difference in the image's pixel values between any given odd and even frames as a result of these moving red cells. Using the imaging program, identical pixel values between the odd and even frames were removed, leaving behind only pixels that changed from frame to frame-essentially, those pixels depicting red cell movement. The resulting display of the dynamic pixels shows the distance traveled by a red blood cell during the acquisition of a particular odd and even frame (a shifting vector). This shifting vector not only can be used to measure the velocity of the red blood cells of the zebrafish larvae but also can be summed to form an aggregate image that ultimately outlines the complete vascular system of the zebrafish. This aggregate essentially provides a "digital cast" of the vascular system in all vessels transited by red blood cells (Fig. 2A). Vessel diameters and blood distribution can then be extracted from these casts.

\section{Statistics}

A two-way repeated-measures ANOVA was performed to assess differences in mean vessel diameter at different viewing angles (the repeated measure) within each larva and during development in different larvae. A one-way ANOVA was used to assess mean differences in red blood cell velocity, heart rate, stroke volume, and cardiac output during development. When comparing the two-dimensional calculation of cardiac output with the three-dimensional calculation during development, a two-way ANOVA was performed on the calculated values of cardiac output and on the residuals. When data lacked a normal distribution or equal variance, an appropriate nonparametric test was performed. Tukey's multiple comparison procedure (Dunn's multiple comparison procedure for nonparametric analyses) was performed post hoc on those groups with significant effects or interactions. All tests were performed using SigmaStat 2.0 (SPSS), and the alpha level was set at $P<0.05$. All results shown are the mean \pm SEM unless otherwise indicated.

\section{Results}

\section{Vessel Size and Development}

Cross-sectional areas of vessels in situ constructed from twodimensional views at five different angles are presented in Figure 2. At all three measurement locations along the zebrafish tail (landmarks shown in Fig. 2), the vein always had a significantly higher cross-sectional area than the corresponding artery. A very abrupt and significant decrease in cross-sectional area was noted during the transition from dorsal artery to caudal artery, with a corresponding increase from caudal vein to dorsal vein.

Interestingly, there was a significant age effect $(P<0.001)$ in all vessels, as cross-sectional area significantly decreased (as much as a $64 \%$ decrease in the case of the dorsal vein) as time progressed from day 2 to day 5 (ascending up the $Y$-axis in Fig. 2). There were no significant differences in vessel diameter within each viewing angle except for the caudal section of the 
A

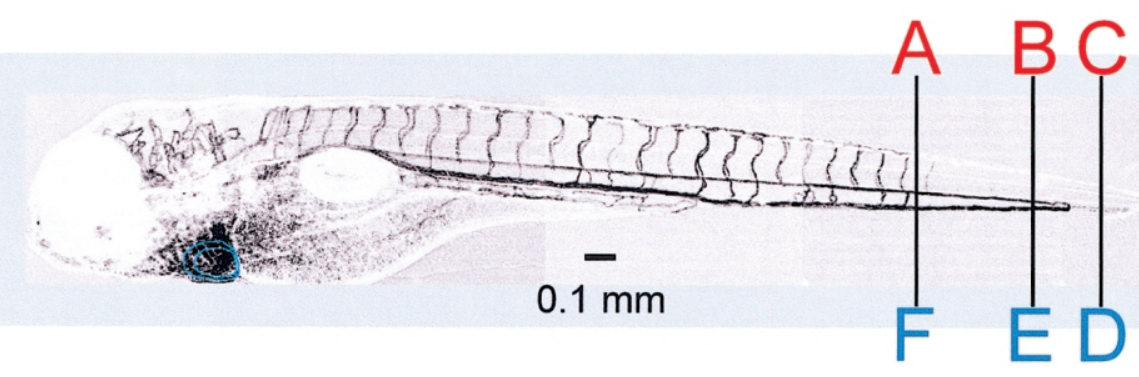

B

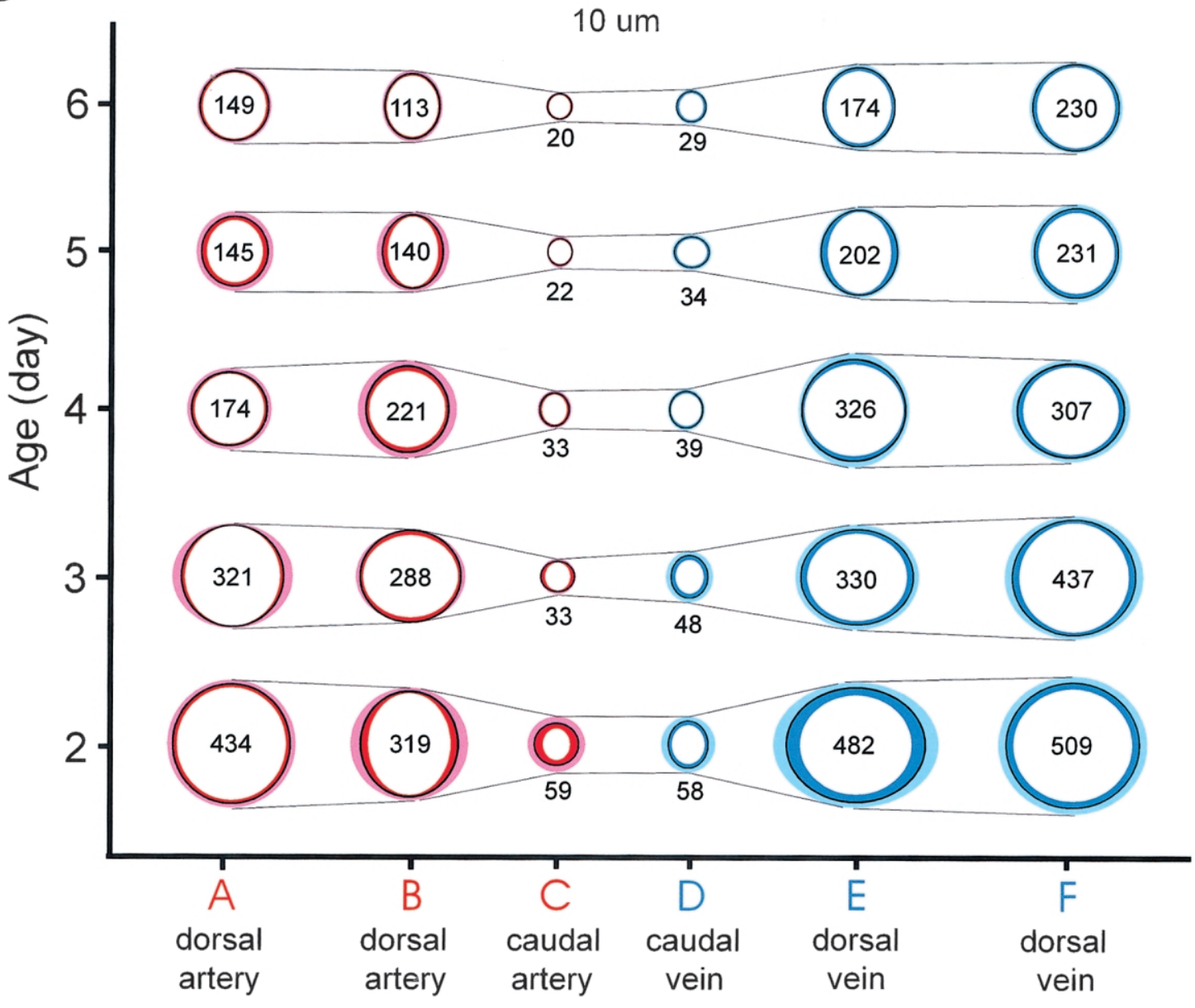

Figure 2. A, Diagram indicating the anatomical positions in posterior zebrafish larvae where cross-sectional area of major arteries and veins was measured during early development. The lines $A-F, B-E$, and $C-D$ indicate vessel measurements at transects between the twenty-fourth and twenty-fifth intersegmental vessels, between the twenty-eighth and twenty-ninth intersegmental vessels, and at a vessel midway in the caudal loop, respectively. $B$, Images of mean cross-sectional area of major arteries and veins during early development. The black outline indicates the mean vessel cross section, while the surrounding color shading indicates the standard error of the mean. Numerical values indicate the corresponding vessel cross-sectional area $\left(\mu \mathrm{m}^{2}\right) . N=8$ for each angle of each vessel on each day. 


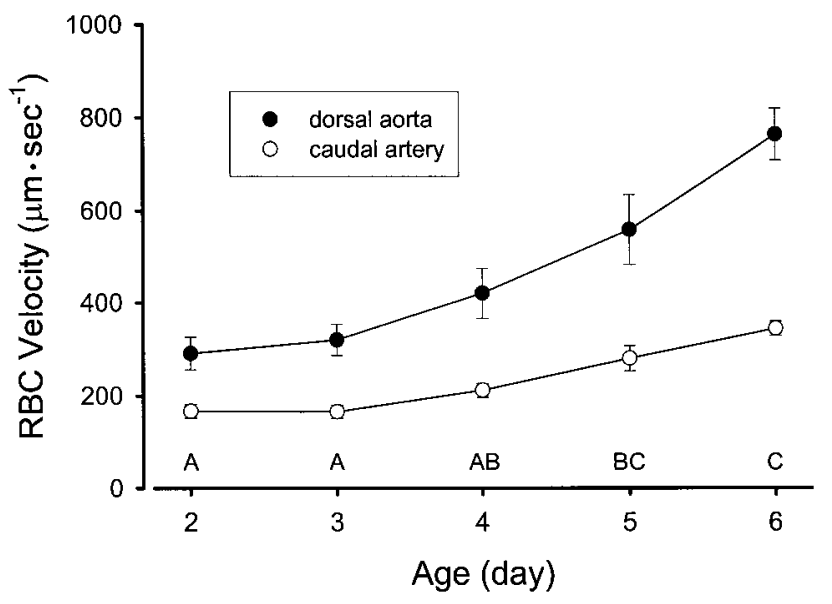

Figure 3. Mean red blood cell velocity through the caudal artery and the dorsal artery of zebrafish larvae during early development. Letter groupings indicate relationships of mean values during development as calculated with post hoc tests. $N=6$ for days 2,5 , and $6 . N=7$ for days 3 and 4 .

dorsal vein ( $E$ in Fig. $2 ; F=4.7, P=0.002$ ). This indicates that early in development, the dorsal vein was significantly dorsoventrally compressed (i.e., wider than high), a transient feature that disappeared with further development. Additionally, vessel boundaries became more morphologically distinct throughout this 5-d developmental period, as is evident in the reduced standard error of the mean cross-sectional area.

\section{Red Blood Cell Velocities}

Red blood cell velocity in the dorsal artery significantly increased during early development $(F=18.9, P<0.001)$, from $291 \pm 35 \mu \mathrm{m} \mathrm{s}^{-1}$ at day 2 to $766 \pm 55 \mu \mathrm{m} \mathrm{s}^{-1}$ at day 6 (Fig. $3)$. As development progressed, red blood cell velocity profiles within each individual zebrafish larva did not vary significantly in the caudal artery $(F=2.6, P=0.056)$. In the dorsal artery, however, the variation in red blood cell velocity within each individual significantly increased during development $(F=$ $3.1, P=0.031)$ as a greater pulsatile movement of red cells was detected.

\section{Cardiac Output}

Significant increases during development occurred in both enddiastolic volume $(F=4.9, P=0.004)$ and end-systolic volume $(F=3.7, P=0.015$; Fig. 4). End-diastolic volume increased proportionally more than end-systolic volume. This resulted in a significant increase $(F=5.0, P=0.004)$ in stroke volume during development, from $0.146 \pm 0.016 \mathrm{~nL}$ at day 2 to $0.297 \pm 0.040 \mathrm{~nL}$ at day 6 (Fig. $5 B$ ). Additionally, the twofold increase in stroke volume during the first $5 \mathrm{~d}$ of development, coupled with a significant increase $(F=27.0, P<0.001)$ in heart rate over this period, from $111 \pm 5$ to $169 \pm 5$ beats $\min ^{-1}$ (Fig. $5 A$ ), resulted in a sixfold increase in cardiac output (Fig. 6).

Two-dimensional calculation of cardiac output, assuming the heart to be shaped as a prolate spheroid, revealed an increase from $9.3 \pm 1.5 \mathrm{~nL} \mathrm{~min}{ }^{-1}$ at day 2 to $55.6 \pm 15.5 \mathrm{~nL} \mathrm{~min}{ }^{-1}$ at day 6 of development (Kruskal-Wallace ANOVA on ranks $H=9.5, P<0.05$; Fig. $6 A$ ). However, when the three-dimensional measurement was added and equation (2) used to calculate stroke volume in the same larva, a significantly higher cardiac output was recorded $(F=9.3, P<0.001)$, as were significantly lower statistical residuals $(F=10.9, P=0.002$; Fig. $6 B)$.

\section{Discussion}

\section{Critiques of the Techniques}

More advanced techniques exist for imaging zebrafish in three dimensions, such as confocal microscopy with associated digital particle image velocimetry (DPIV; Hove et al. 2003). Additionally, an online data set from the Weinstein lab posts a more precise measurement of vessel diameter and heart shape in three dimensions (http://dir.nichd.nih.gov/lmg/uvo/atlas.html). However, we have presented a technique for measuring both cardiac and vessel size in three dimensions using conventional light microscopy. Not only is this technique less costly, but, more important, it also allows the measurement of cardiovascular parameters in vivo, a prerequisite for studying cardiovascular responses to pharmacological agents.

The heart of the zebrafish larva starts beating at approximately $24 \mathrm{hpf}$ at $28.5^{\circ} \mathrm{C}$. During this early organization phase, the heart is somewhat irregularly shaped, especially when viewed from the lateral side. In fact, it does not take its rec-

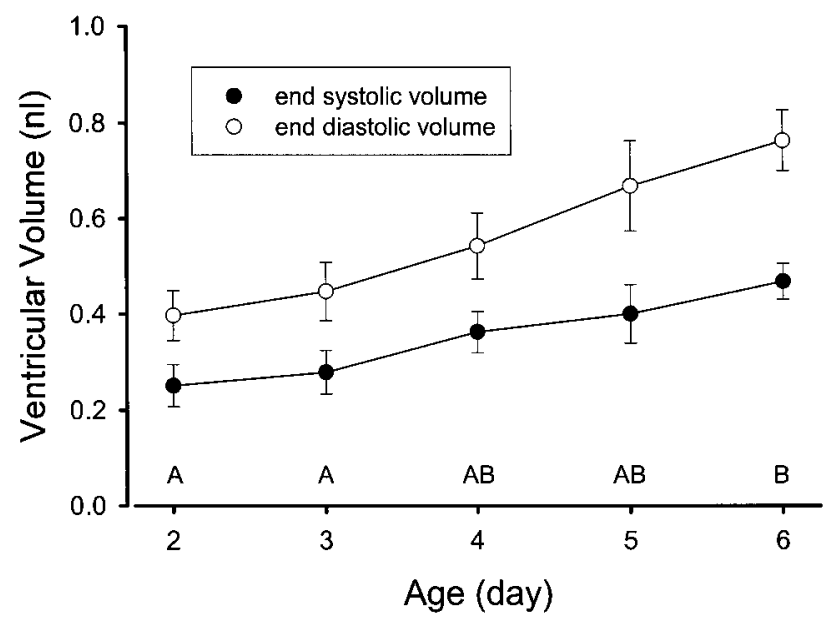

Figure 4. Mean end-diastolic and end-systolic volume in zebrafish larvae hearts during early development. Letter groupings indicate statistical relationships of mean values during development and are identical for both sets of vessel data. $N$ values are as in Figure 3 . 
A

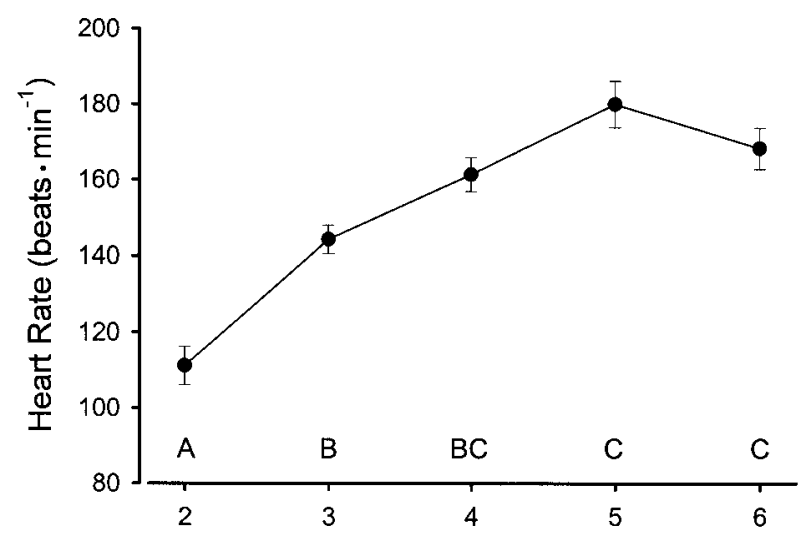

B

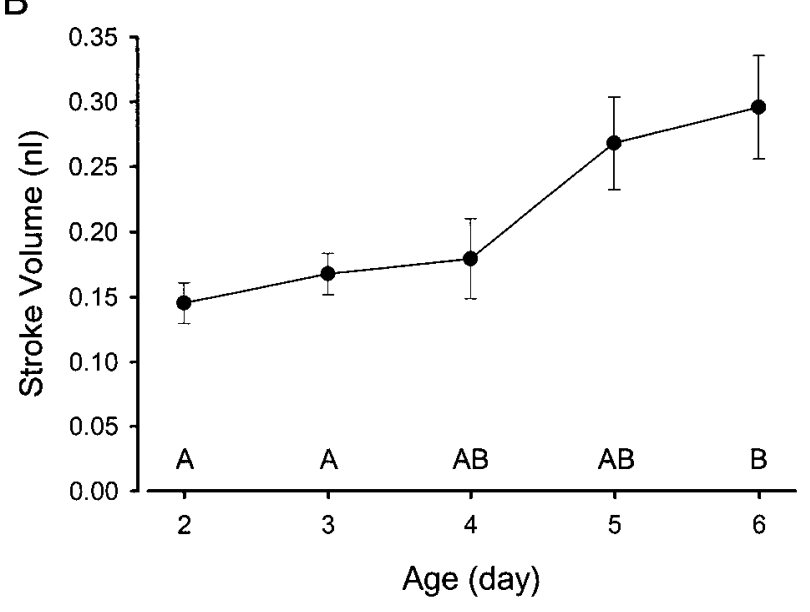

Figure 5. Heart rate $(A)$ and stroke volume $(B)$ in zebrafish larvae during early development. Letter groupings indicate statistical relationships between mean values during development. $N$ values are as in Figure 3.

ognizable adult shape of a prolate spheroid until $5 \mathrm{~d}$ postfertilization at this temperature (Weinstein and Fishman 1996). Yet, most common previous studies for visual measurement of cardiac output in vertebrate embryos have used the equation for a prolate spheroid for modeling heart shape throughout early development (Burggren and Fritsche 1995; Hou and Burggren 1995; Burggren 2004). The observations of heart shape in this study suggest that the use of a prolate spheroid leads to less accurate stroke volume measurements before day 5 in the zebrafish at $28.5^{\circ} \mathrm{C}$ (day 6 at $25^{\circ} \mathrm{C}$ ). Furthermore, the equation for the volume of a prolate spheroid assumes that the ventral width of the heart is identical to its lateral height, and so the measurements used in its calculation are two radii. All of these variables combine to give highly variable and potentially inaccurate representations of stroke volume and thus cardiac output (Figs. 5, 6).

By modifying the technique used by Hou and Burggren
(1995) and updated by Schonweger et al. (2000) and Schwerte and Pelster (2000), we have considerably improved the stroke volume measurements and thus cardiac output calculations, as shown by the significant reduction in variability from Figure $6 A$ to $6 B$. This technique now allows a more powerful detection of dimensional differences when studying treatment effects, for example. Additionally, imaging the beating heart on the ventral as well as both lateral sides results in a more powerful assessment of the dimensional changes of the beating heart during development. Instead of using two radii as the equation variables, tracing the ventricular area in the video images and inserting this into equation (2) produces a cardiac volume more representative of the true shape of the heart, especially in the earliest developmental stages. Notably, while the threedimensional method described in this study is particularly effective in early stages, it may not be substantially superior in

\section{A}

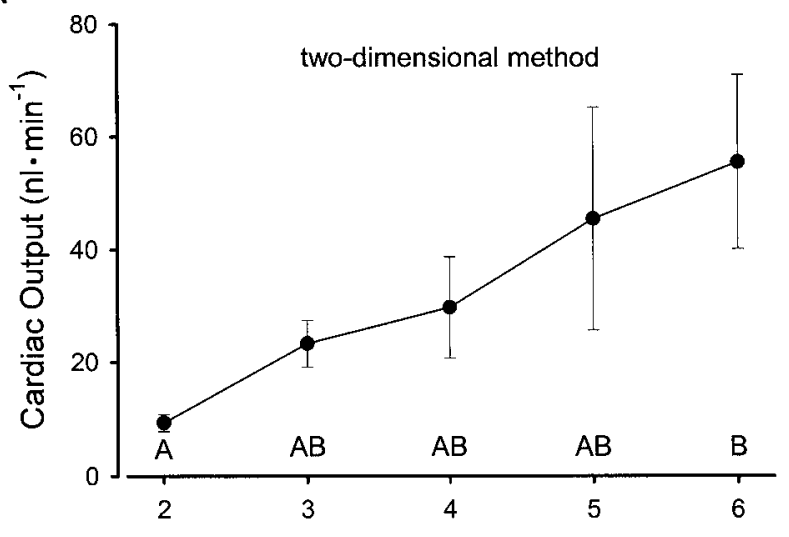

B

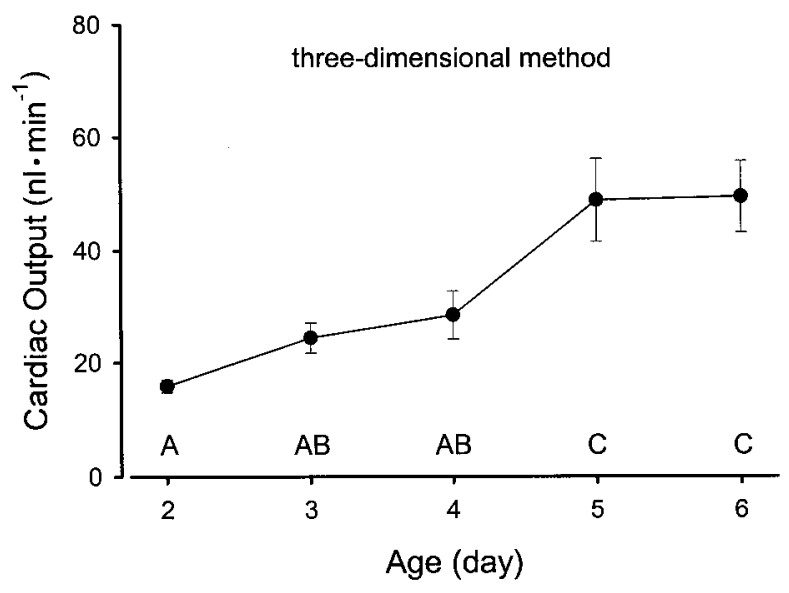

Figure 6. Mean cardiac output calculated by equation (1) using a twodimensional analysis $(A)$ and calculated by equation (2) using three dimensions $(B)$. Letter groupings indicate statistical relationships of mean values during development and are identical for both sets of vessel data. $N$ values are as in Figure 3. 
older stages where the larval heart has reached an adultlike form (Fig. 6).

\section{Cardiac Changes during Early Development}

Days 5 and 6 show the most striking changes of the cardiovascular system during the early developmental period. Vessel diameters decrease, and red blood cell velocities, heart rate, and stroke volume all increase, the latter changes contributing to an increased cardiac output. These changes could arise from a suite of anatomical and physiological adjustments. The transition of the larval heart to an adultlike form (by day 5 at $28.5^{\circ} \mathrm{C}$; Weinstein and Fishman 1996) appears to be accompanied by increased effectiveness, with not only more blood pumped per stroke but also an increased red cell velocity. Additionally, the cross-sectional area of the vascular beds increases as peripheral vessels proliferate, leading to a distinct fall in peripheral resistance (Hou and Burggren 1995). These rapid changes in heart performance, especially cardiac output, are concurrent with the transition from dependence on direct transcutaneous $\mathrm{O}_{2}$ uptake to branchial $\mathrm{O}_{2}$ uptake and the need for internal blood convection via the cardiovascular system (Pelster and Burggren 1996). This transition from diffusive to convective $\mathrm{O}_{2}$ demands large increases in cardiac output, even though the heart has already been beating for days, which likely contributes to angiogenesis rather than gas exchange (Burggren 2004).

\section{Vascular Changes in Early Development}

Assessing the in vivo cross section of the anteriocaudal vessels in fish larvae can be quite difficult, since vessels may collapse quite easily during standard methods for tissue fixing, sectioning, and staining. Moreover, typical optical techniques do not account for the fact that some vessels may not have perfectly round cross sections. Consequently, we modified Schwerte and Pelster's (2000) technique to view vessels in vivo through $180^{\circ}$ rotation during the first $6 \mathrm{~d}$ of development (Fig. 2). Interestingly, mean vessel cross-sectional area significantly decreased over this period of early development, a counterintuitive finding, yet one in accordance with the manner in which blood vessels form in the early zebrafish larva. The overall form of the vasculature is established early, before the initiation of circulation, and the pattern of vessel tracts is reproducible from embryo to embryo (Isogai et al. 2003). In the zebrafish, cells from the dorsal margin of the lateral plate migrate medially to form the intermediate cell mass (Weinstein and Fishman 1996). This mass gives rise to both the endothelia of the major trunk vessels and the first cohort of blood cells (Childs et al. 2002). However, many early vasculogenic vessels first appear as a network or plexus of smaller vessels, with little apparent pattern or differentiated identity (Weinstein 1999, 2002). The final pattern and identity of vessels emerge only upon angiogenic re- modeling of these primary networks, and the particular final pattern formed is plastic and easily altered by changes in the local environment (Weinstein and Fishman 1996; Yelon 2001; Rossant and Hirashima 2003).

Our observations clearly support this proposed model for vascular remodeling during early development. On days 2 and 3 , venous blood returning to the heart followed several distinct but closely parallel paths along the larva's longitudinal axis. As the larva developed a more defined network of major vessels, not only did visual density of red blood cells increase in a particular vessel length, but the velocity was also significantly higher (Fig. 3). It was also clear that once the final and distinct vessel pattern was established, vessel growth (as measured by increase in vessel diameter) slowed considerably thereafter. In a study using the DPIV technique, lower limits of intracardiac red blood cell velocities at $4.5 \mathrm{~d}$ postfertilization were approximately $0.5 \mathrm{~cm} \mathrm{~s}^{-1}$, and upper limits were $1.0 \mathrm{~cm} \mathrm{~s}^{-1}$ (Hove et al. 2003). With intracardiac velocities being higher than vessel velocities, our data fit well within the range of the study by Hove et al. (2003).

In summary, the three-dimensional technique described here provides accurate dimensional measurement of cardiovascular structure. Using this technique, we have elucidated further detail in the early physiology of the developing zebrafish larvae. Profound changes in the cardiovascular system occur especially during days 5 and 6 of development, leading to enhanced cardiac output and presumably enhanced nutrient delivery to service growing tissues. Further experiments using this technique will address underlying mechanisms of early cardiovascular development.

\section{Acknowledgments}

We thank Dr. Bernd Pelster and Dr. Thorsten Schwerte for their help in learning physiological imaging techniques. We also thank Luke Woloszyn for his assistance in data gathering. This project was supported by National Science Foundation grant IBN-0128043.

\section{Literature Cited}

Bagatto B. 2005. Ontogeny of cardiovascular control in zebrafish (Danio rerio): effects of developmental environment. Comp Biochem Physiol A 141:391-400.

Bagatto B., B. Pelster, and W.W. Burggren. 2001. Growth and metabolism of larval zebrafish: effects of swim training. J Exp Biol 204:4335-4343.

Burggren W.W. 2004. What is the purpose of the embryonic heart beat? or how facts can ultimately prevail over physiological dogma. Physiol Biochem Zool 77:333-345.

Burggren W.W. and R. Fritsche. 1995. Cardiovascular mea- 
surements in animals in the milligram body mass range. Braz J Med Biol Res 28:1291-1305.

Childs S., J.N. Chen, D.M. Garrity, and M.C. Fishman. 2002. Patterning of angiogenesis in the zebrafish embryo. Development 129:973-982.

Fishman M.C. and K.R. Chien. 1997. Fashioning the vertebrate heart: earliest embryonic decisions. Development 124:20992117.

Fritsche R. and W.W. Burggren. 1996. Development of cardiovascular responses to hypoxia in the larvae of the frog Xenopus laevis. Am J Physiol 271:R912-R917.

Glickman N.S. and D. Yelon. 2002. Cardiac development in zebrafish: coordination of form and function. Semin Cell Dev Biol 13:507-513.

Hou P.L. and W.W. Burggren. 1995. Blood pressures and heart rate during larval development in the anuran amphibian Xenopus laevis. Am J Physiol 269:R1120-R1125.

Hove J.R., R.W. Köster, A.S. Forouhar, G. Acevedo-Bolton, S.E. Fraser, and M. Gharib. 2003. Intracardiac fluid forces are an essential epigenetic factor for embryonic cardiogenesis. $\mathrm{Na}-$ ture 421:172-177.

Isogai S., N.D. Lawson, S. Torrealday, M. Horiguchi, and B.M. Weinstein. 2003. Angiogenic network formation in the developing vertebrate trunk. Development 130:5281-5290.

Mirkovic T. and P. Rombough. 1998. The effect of body mass and temperature on heart rate, stroke volume, and cardiac output of larvae of the rainbow trout, Oncorhynchus mykiss. Physiol Zool 71:191-197.

Pelster B. and W.W. Burggren. 1996. Disruption of hemoglobin oxygen transport does not impact oxygen-dependent phys- iological processes in developing embryos of zebra fish ( $D a$ nio rerio). Circ Res 79:358-362.

Rossant J. and M. Hirashima. 2003. Vascular development and patterning: making the right choices. Curr Opin Genet Dev 13:408-412.

Schonweger G., T. Schwerte, and B. Pelster. 2000. Temperaturedependent development of cardiac activity in unrestrained larvae of the minnow Phoxinus phoxinus. Am J Physiol 279: R1634-R1640.

Schwerte T. and R. Fritsche. 2003. Understanding cardiovascular physiology in zebrafish and Xenopus larvae: the use of microtechniques. Comp Biochem Physiol A 135:131-145.

Schwerte T. and B. Pelster. 2000. Digital motion analysis as a tool for analyzing the shape and performance of the circulatory system in transparent animals. J Exp Biol 203:16591669.

Stainier D.Y.R. and M.C. Fishman. 1992. Patterning the zebrafish heart tube: acquisition of anteroposterior polarity. Dev Biol 153:91-101.

Weinstein B.M. 1999. What guides early embryonic blood vessel formation? Dev Dyn 215:2-11.

- 2002. Plumbing the mysteries of vascular development using the zebrafish. Semin Cell Dev Biol 13:515-522.

Weinstein B.M. and M.C. Fishman. 1996. Cardiovascular morphogenesis in zebrafish. Cardiovasc Res 31:E17-E24.

Westerfield M. 1995. The Zebrafish Book. University of Oregon Press, Eugene.

Yelon D. 2001. Cardiac patterning and morphogenesis in zebrafish. Dev Dyn 222:552-563. 
Copyright of Physiological \& Biochemical Zoology is the property of University of Chicago Press and its content may not be copied or emailed to multiple sites or posted to a listserv without the copyright holder's express written permission. However, users may print, download, or email articles for individual use. 